\title{
Coherent and incoherent radiation from free-electron lasers with an axial guide field
}

\author{
H. P. Freund* and P. Sprangle \\ Naval Research Laboratory, Washington, D. C. 20375 \\ D. Dillenburg, E. H. da Jornada, B. Liberman, and R. S. Schneider \\ Instituto de Fisica, Universidade Federal do Rio Grande do Sul, 90.000 Porto Alegre-RS, Brazil
}

(Received 27 March 1981)

\begin{abstract}
The spontaneous and induced emission from a free-electron laser is treated for the case in which an axial magnetic field is imposed in addition to the helical, axially periodic wiggler magnetic field. The classes of possible single-particle trajectories in this configuration are discussed, and the results are applied to a calculation of the incoherent radiation from a beam of relativistic electrons in the system. The coherent radiation is treated by solving the Vlasov-Maxwell equations for the linear gain in the tenuous-beam limit, where the beam plasma frequency is much less than the radiation frequency and self-field effects can be ignored.
\end{abstract}

\section{INTRODUCTION}

Recent experiments ${ }^{1-3}$ have amply demonstrated the usefulness of the free-electron laser as a tunable source of coherent submillimeter radiation, and have stimulated interest in a variety of experimental configurations. Theoretical work ${ }^{4-6}$ in this regard has concentrated on the case in which a relatively low-density (i.e., self-field effects are negligible), relativistic electron beam is propagated through a periodic helically symmetric magnetic field, referred to as the "wiggler" field. In the beam frame the wiggler field appears as a backwards propagating electromagnetic wave, and radiation is produced by means of coherent scattering of this wave off electrostatic fluctuations in the beam. Another configuration employed is one in which an axial guide field is present, and is used principally, but not exclusively, in the regime in which electron densities and currents are sufficiently high that the axial field is required to contain the beam against the effects of the self-fields. Theoretical analyses of the coherent radiation mechanism in this regime have begun to appear in the literature for this configuration as well. ${ }^{7-11}$

It is our purpose in this work to treat both the coherent and incoherent radiation from a freeelectron-laser configuration which contains an axial guide field in the low-density limit. Full relativistic effects are included, but the self-fields of the beam are neglected. The principal application of this study to existing experiments is the Stanford University free-electron laser ${ }^{1,2}$ in which a low - current ( 2-A), high-energy ( $24-43-\mathrm{MeV})$ beam was injected into a drift tube in which an axial guide field of $1 \mathrm{kG}$ and a wiggler field of about 2.4 $\mathrm{kG}$ were applied. In this regime collective beam effects are negligible.

The organization of the paper is as follows. In Sec. II, we discuss the single-particle trajectories of electrons in a magnetic field geometry composed of a combined axial guide field and helical wiggler field. The incoherent, spontaneous emission spectrum is calculated in Sec. III using test-particle techniques, and the linear gain is found in Sec. IV in the limit of a low-density beam. In treating a low-density beam, we restrict the analysis of the linear gain to the small-signal regime but provide a fully kinetic derivation of the gain based upon the Vlasov-Maxwell equations. This is in contrast to the work of Kwan and Dawson ${ }^{7}$ and Bernstein and Friedland ${ }^{10}$ in which collective effects due to high beam density were included in the context of a fluid theory of the interaction. The small-signal gain has also been considered by Friedland and Hirschfield ${ }^{11}$ by means of a fluid analysis. Thus, we provide a fully kinetic expression for the smallsignal gain and an extensive description of the parametric dependence of the gain in the coldbeam limit. A summary and discussion is given in Sec. V.

\section{SINGLE-PARTICLE TRAJECTORIES}

The wiggler field is generally due to a helical current winding and the resultant field can be

1965 (c)1981 The American Physical Society 
shown, in a self-consistent way, to be of the form $^{12,13}$

$$
\overrightarrow{\mathrm{B}}=B_{0} \hat{e}_{z}+\overrightarrow{\mathrm{B}}_{w}(r, z),
$$

where the wiggler field is derived from a vector potential of the form

$$
\begin{aligned}
\overrightarrow{\mathbf{A}}_{w}=-\frac{2 B_{w}}{k_{w}} & \frac{1}{k_{w} r} I_{1}\left(k_{w} r\right) \cos \left(\theta-k_{w} z\right) \hat{e}_{r} \\
& \left.-I_{1}^{\prime}\left(k_{w} r\right) \sin \left(\theta-k_{w} z\right) \hat{e}_{\theta}\right],
\end{aligned}
$$

in cylindrical coordinates. In Eq. (2), $B_{w}$ and $k_{w}$ ( $\equiv 2 \pi / \lambda_{w}$, where $\lambda_{w}$ is the wiggler period) are assumed to be constant, and $I_{1}$ and $I_{1}^{\prime}$ are the modified Bessel function of the first kind and its derivative, respectively. In most free-electron-laser experiments, however, the initial beam radius is a small fraction of the wiggler period, and expansion in powers of $k_{w} r$ yields

$$
\overrightarrow{\mathrm{B}}_{w} \simeq B_{w}\left(\hat{e}_{x} \cos k_{w} z+\hat{e}_{y} \sin k_{w} z\right) .
$$

Rather than work in the laboratory frame, we choose to transform to the frame rotating with the wiggler field. To this end we define

$$
\begin{aligned}
& \hat{e}_{1}=\hat{e}_{x} \cos k_{w} z+\hat{e}_{y} \sin k_{w} z, \\
& \hat{e}_{2}=-\hat{e}_{x} \sin k_{w} z+\hat{e}_{y} \cos k_{w} z, \\
& \hat{e}_{3}=\hat{e}_{z},
\end{aligned}
$$

and write the orbit equations corresponding to the field structure given in (1) and (3) in the form

$$
\begin{aligned}
& \dot{v}_{1}=v_{2}\left(k_{w} v_{3}-\Omega_{0}\right), \\
& \dot{v}_{2}=-\Omega_{w} v_{3}-v_{1}\left(k_{w} v_{3}-\Omega_{0}\right), \\
& \dot{v}_{3}=\Omega_{w} v_{2},
\end{aligned}
$$

where $\Omega_{0} \equiv\left|e B_{0} / \gamma m c\right|, \Omega_{w} \equiv\left|e B_{w} / \gamma m c\right|$, and $\gamma \equiv\left(1-v^{2} / c^{2}\right)^{-1 / 2}$. Since the total energy is a conserved quantity, $v^{2} \equiv v_{1}^{2}+v_{2}^{2}+v_{3}^{2}$ is a constant of the motion and Eqs. (4) are fully relativistic. The principal benefit derived from this transformation is that the equations of motion are now coordinate independent, and depend only on the components of the velocity and their derivatives.

In addition to the total energy, a second constant of the motion can be found by elimination of $v_{2}$ from (4); specifically, $u \equiv v_{1}-k_{w}\left(v_{3}-\Omega_{0} / k_{w}\right)^{2} / 2 \Omega_{w}$. This is analogous to the axial invariant discussed by Davidson and Uhm, ${ }^{14}$ which in the $B_{w} \rightarrow 0$ limit implies that the axial velocity is conserved and defines the rotating frame to be that of the Larmor rotation of an electron in a uniform magnetic field. By means of these constants, the problem can be reduced to the solution of a single nonlinear differential equation for $v_{3}$,

$$
\left(\frac{d x}{d \tau}\right)^{2}+\Phi(x)=0,
$$

where

$$
\begin{aligned}
& x \equiv \beta_{3}-\beta_{0}, \quad \tau \equiv \Omega_{0} t / 2 \\
& \beta_{3} \equiv v_{3} / c, \quad \beta_{0} \equiv \Omega_{0} / k_{w} c, \\
& \Phi(x) \equiv x^{4}+4 \epsilon \beta_{0}\left(\epsilon \beta_{0}+\beta_{u}\right) x^{2}+8 \epsilon^{2} \beta_{0}^{3} x \\
& \quad+4 \epsilon^{2} \beta_{0}^{2}\left(\beta_{0}^{2}+\beta_{u}^{2}-\beta_{v}^{2}\right),
\end{aligned}
$$

$\epsilon \equiv B_{w} / B_{0}, \beta_{v} \equiv v / c$, and $\beta_{u} \equiv u / c$. Note that Eq (5) has the trivial solution $x=0$ (i.e., $v_{3}=$ const) in the limit of a uniform axial magnetic field $\left(B_{w} \rightarrow 0\right)$.

A detailed discussion of the solutions of Eq. (5) has been given by Freund and Drobot ${ }^{15}$; however, we restrict our attention here to solutions corresponding to relatively uniform axial velocities. The reason for this is that the radiation mechanism is a resonant one in which the emission frequency is given by $\omega \simeq 2 \gamma_{z}^{2} k_{w} v_{3}$, where $\gamma_{z}=\left(1-v_{3}^{2} / c^{2}\right)^{-1 / 2}$. As a result, variations in the axial velocity of the order of $\Delta v_{3}$ lead to a broadening of the emission spectrum which scales as $\Delta \omega \sim 2 \gamma_{z}^{3} k_{w} \Delta v_{3}$. Thus, while small oscillations in $v_{3}$ about some bulk axial velocity lead to a relatively narrow bandwidth, large oscillations can result in spectral broadening with a corresponding decrease in the linear gain.

The conditions which lead to small oscillations in the axial velocity can be investigated by consideration of the roots of the pseudopotential $\Phi(x)$. Physically meaningful (i.e., real) solutions are possible only when $\Phi<0$; therefore, the real roots of the pseudopotential correspond to the bounds on the oscillation about some bulk axial velocity (defined by the local minimum in $\Phi$ between the roots). The typical character of the pseudopotential when $\epsilon<1$ and $\beta_{0}>1$ is shown in Fig. 1. In this regime, the pseudopotential has two real roots which for given values of $\epsilon, \beta_{0}$, and $v$ shift in both position and spacing with variations in $\beta_{u}$ within some fixed range. The extrema in the range of $\beta_{u}$ occur when the real roots of $\Phi$ are degenerate, and correspond to solutions with constant axial veloci$\mathrm{ty}^{15,16}$

$$
\begin{aligned}
& v_{1}=-\frac{\Omega_{w} v_{\|}}{k_{w} v_{\|}-\Omega_{0}}, \\
& v_{2}=0, \\
& v_{3}=v_{\|},
\end{aligned}
$$




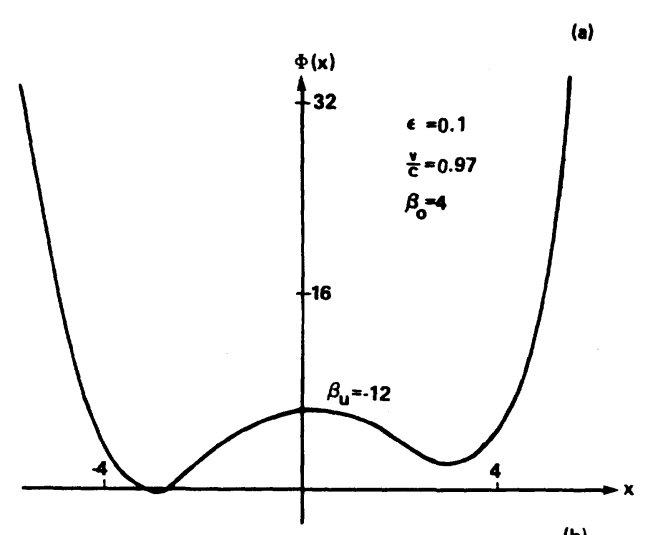

(b)

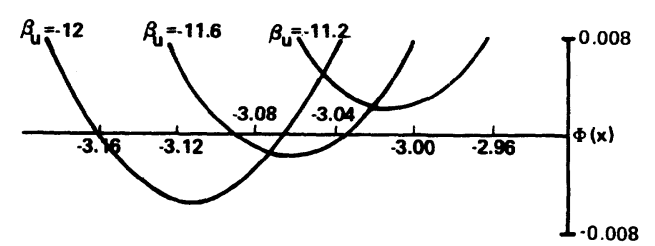

FIG. 1. Graph of the pseudopotential $\Phi$ versus $x$ for $\epsilon=0.1, v / c=0.97$, and several values of $\beta_{0}$.

where the axial velocity $v_{\|}$is given by $v^{2}=v_{1}^{2}+v_{3}^{2}$ for some choice of the total energy.

The values of $\beta_{3}\left(=\beta_{0}+x\right)$ corresponding to the real roots of $\Phi$ are shown in Fig. 2 versus $\beta_{u}$ for $\epsilon=0.1$, and $\beta_{0}=1$ and 4 . Thus, the figure describes the bounds on the oscillations in the axial velocity for given $\beta_{u}$. It is important to recognize that small deviations in $\beta_{u}$ from the extrema result in trajectories which do not differ greatly from

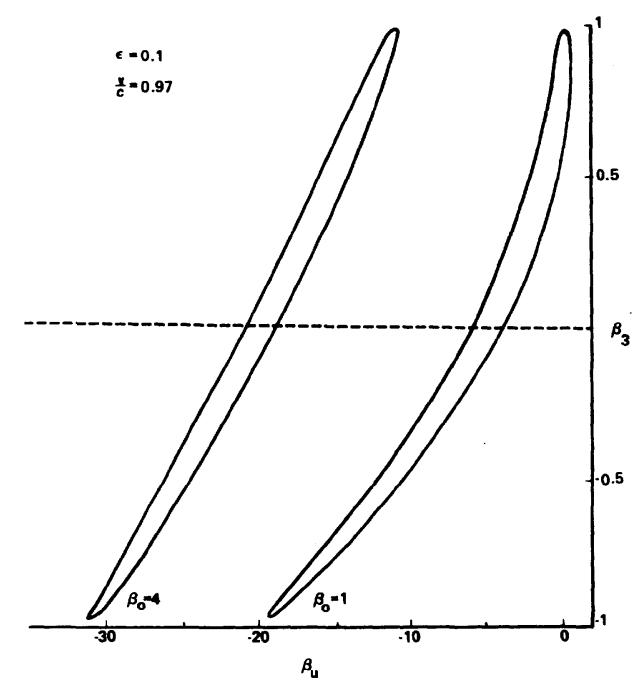

FIG. 2. Graph of the bounds on the axial velocity versus $\beta_{u}$ for $\epsilon=0.1, v / c=0.97$, and $\beta_{0}=1,4$. those with uniform axial velocities. Because of this, two electrons which are characterized by only slightly different values of $\beta_{u}$ and which are close together at some point will not undergo a large separation in the course of their orbits. To be more specific, an initially bunched electron beam will tend to remain bunched. This describes an "orbitally stable" uniform- $v_{3}$ solution, in the sense discussed by Friedland. ${ }^{16}$ A quantitative test for orbital stability can be expressed in the form

$$
\frac{\epsilon^{2} \beta_{0}^{3}}{\left(v_{\|} / c-\beta_{0}\right)^{3}}<1,
$$

which (since $v_{\|}<c$ ) is trivially satisfied for the uniform $-v_{3}$ trajectories when $\beta_{0}>1$.

In contrast, when $\beta_{0}$ is less than unity the pseudopotential can have as many as four real roots, and as many as four uniform- $v_{3}$ orbits appear. An example of this is shown in Fig. 3, where we plot $\beta_{3}$ corresponding to the roots versus $\beta_{u}$ for $\epsilon=0.1$, and $\beta_{0}=0.5$ and 1 . The orbits with uniform axial velocity correspond to the points of vertical slope in the figure, and when $\beta_{0}=0.5$, four such trajectories appear. Three of these orbits are stable; however, the trajectory corresponding to point $A$ in the figure represents a fundamentally different class of solutions than the other three. The behavior of the pseudopotential for values of $\beta_{u}$ in the vicinity of a stable orbit is shown in Fig. 1. In contrast, the behavior of $\Phi$ with $\beta_{u}$ in the vicinity of point $A$ is shown in Fig. 4 in which the uniform- $v_{3}$ trajectory is obtained when the central maximum is zero. Arbitrarily small deviations from this condi-

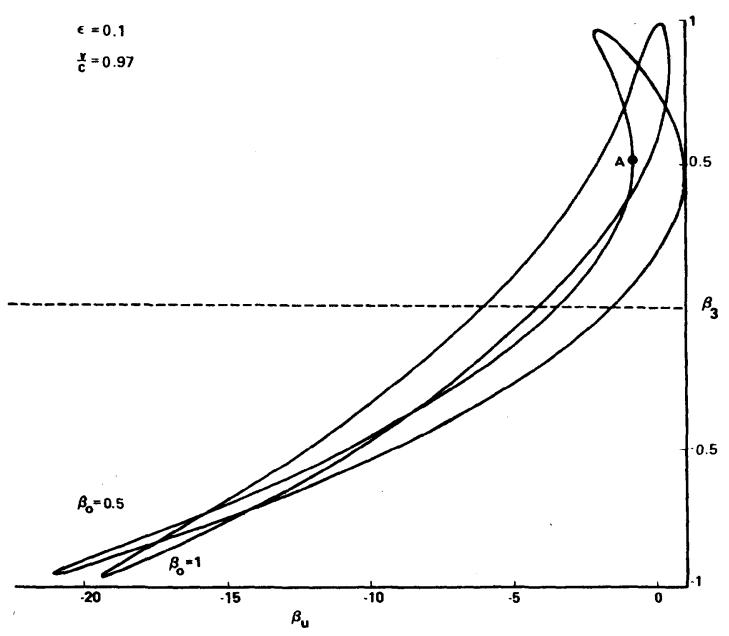

FIG. 3. Graph of the bounds on the axial velocity versus $\beta_{u}$ for $\epsilon=0.1, v / c=0.97$, and $\beta_{0}=0.5,1$. 


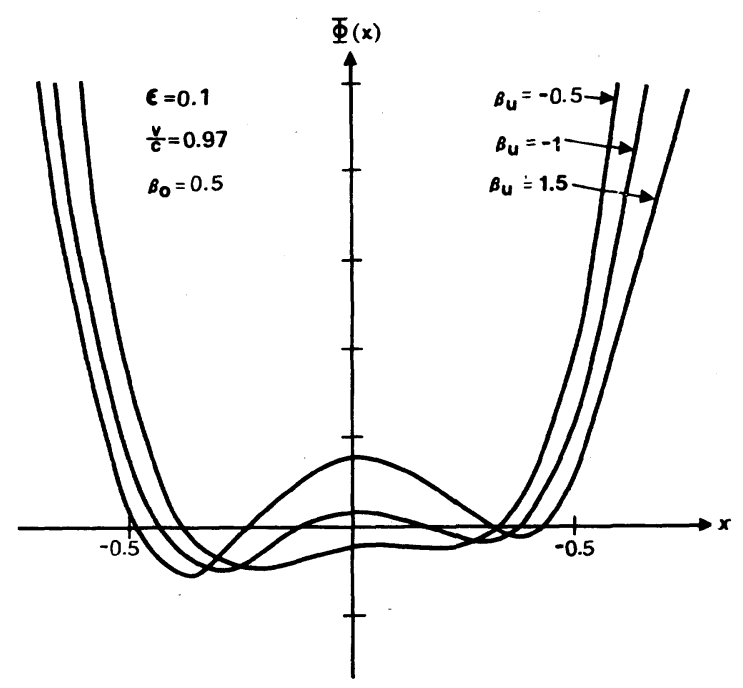

FIG. 4. Graph showing the variation of the pseudopotential with $\beta_{u}$ in the vicinity of an orbitally unstable uniform- $v_{3}$ trajectory.

tion result in drastically different types of trajectory with large fluctuations in the axial velocity. Such uniform- $v_{3}$ trajectories are referred to as being "orbitally unstable," since a bunched electron beam with a spread in the axial invariant in the vicinity of point $A$ will rapidly disperse.
As $\beta_{0}$ decreases further, the range of $\beta_{u}$ in which $\Phi$ possesses four real roots increases, as shown in Fig. 5 for $\beta_{0}=0.1$. In particular, the high axial velocity, uniform- $v_{3}$ trajectory for motion parallel to $\vec{B}_{0}$ becomes more pronounced as $\beta_{0}$ decreases further relative to $\epsilon$. This characteristic of the solutions in the small- $\beta_{0}$ regime exists even where $\epsilon>1$ (see Fig. 6). Finally, we conclude that the requirement that electrons propagate with relatively small fluctuations in axial velocity is most difficult to satisfy when $\beta_{0} \sim 1$. The natural corollary, therefore, that extremely broadbanded emission will occur in this regime is supported by observation. ${ }^{17,18}$

In order to treat cases of nearly uniform axial velocity we implicitly restrict consideration to trajectories corresponding to the stable uniform- $v_{3}$ solutions. In this limit, we can write approximate solutions for the momenta in the laboratory frame as

$$
\begin{aligned}
& p_{x}=\frac{\Omega_{w} p_{\|}}{\Omega_{0}-k_{w} v_{\|}} \cos k_{w} z+P_{x} \cos \Omega_{0} t-P_{y} \sin \Omega_{0} t, \\
& p_{y}=\frac{\Omega_{w} p_{\|}}{\Omega_{0}-k_{w} v_{\|}} \sin k_{w} z+P_{x} \sin \Omega_{0} t+P_{y} \cos \Omega_{0} t, \\
& p_{z}=p_{\|}-\frac{\Omega_{w}}{\Omega_{0}-k_{w} v_{\|}}\left[\begin{array}{l}
\text { (7) } \\
\left.-P_{y} \sin \left(k_{w} z-\Omega_{0} t\right)\right],
\end{array}\right.
\end{aligned}
$$

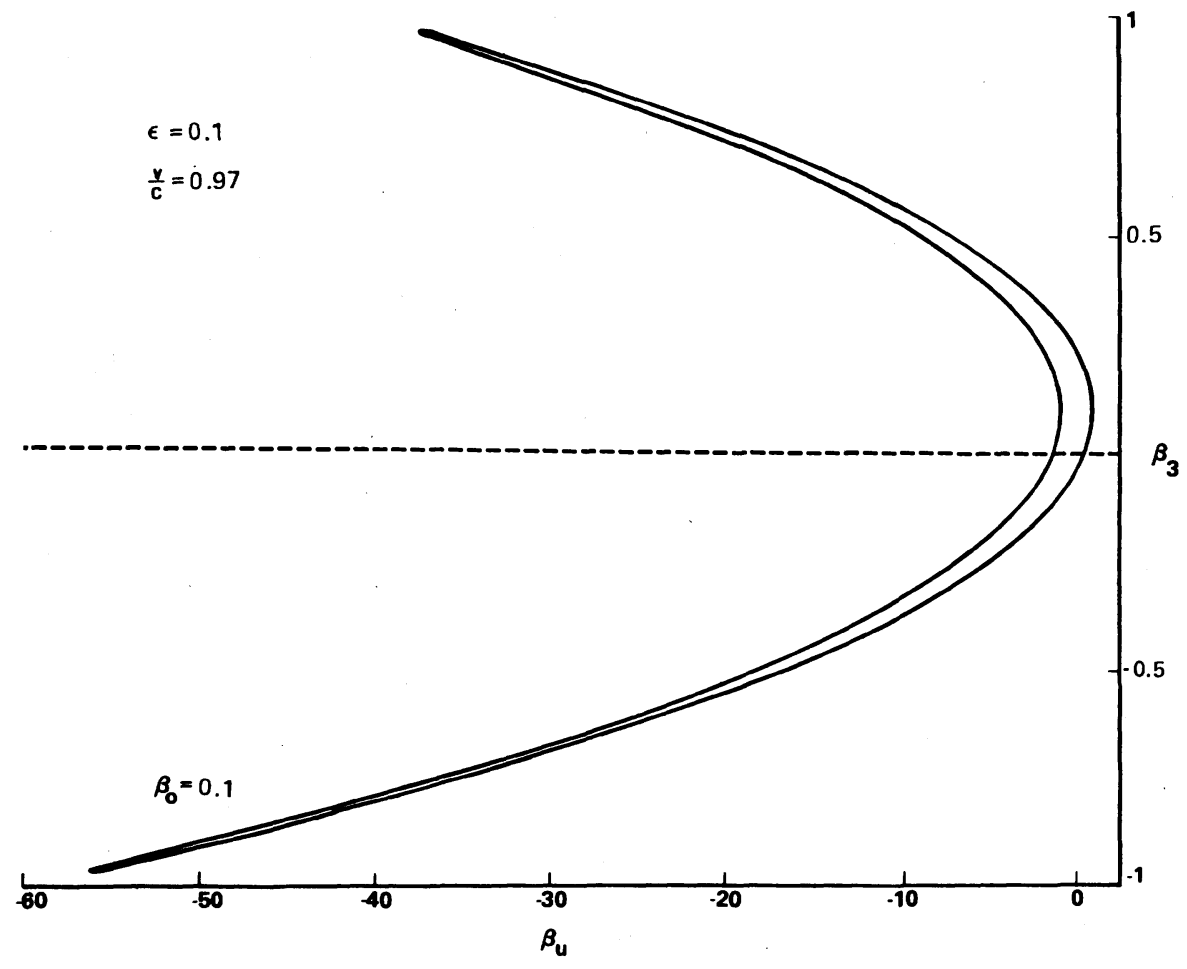

FIG. 5. Graph of the bounds on the axial velocity versus $\beta_{u}$ for $\epsilon=0.1, v / c=0.97$, and $\beta_{0}=0.1$. 


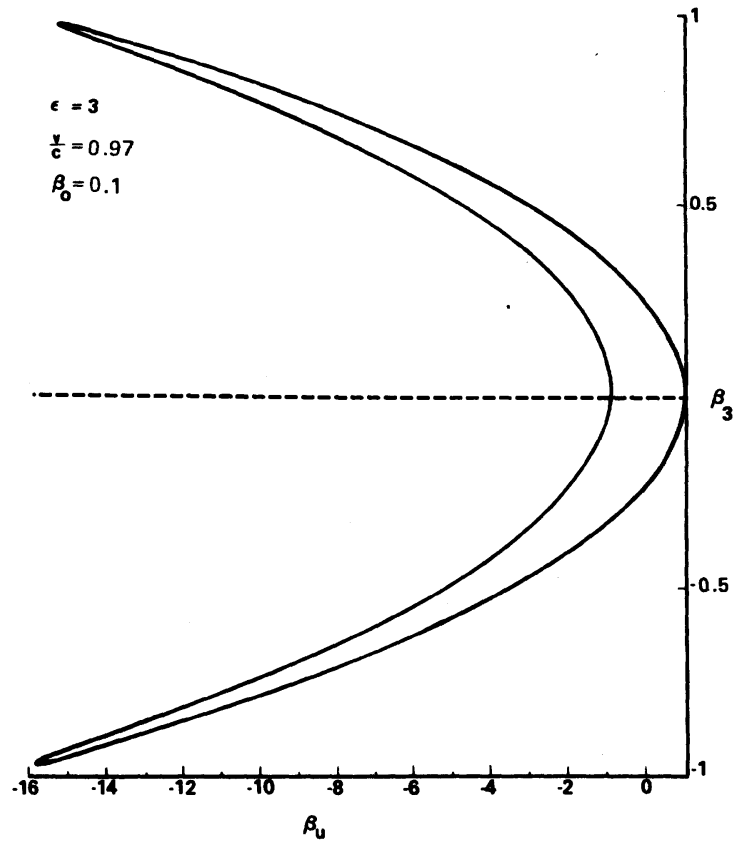

FIG. 6. Graph of the bounds on the axial velocity versus $\beta_{u}$ for $\epsilon=3, v / c=0.97$, and $\beta_{0}=0.01$.

where $p_{\|}, P_{x}$, and $P_{y}$ are approximate constants of the motion, and $v_{\|} \equiv p_{\|} / \gamma m$. Observe that in the limit of a vanishing axial guide field, Eqs. (7) recover the well-known result in which $P_{x}$ and $P_{y}$ are the canonical momenta in the transverse direction. This approximation remains valid as long as $P_{x}^{2}+P_{y}^{2}<<p_{\|}^{2}$, and the total momentum is given by

$$
p^{2}=P_{x}^{2}+P_{y}^{2}+\left(1+\frac{\Omega_{w}^{2}}{\left(\Omega_{0}-k_{w} v_{\|}\right)^{2}}\right) p_{\|}^{2}
$$

to within terms of order $\epsilon^{2}$. The form of these trajectories is shown schematically in Fig. 7. Particle motion predominantly follows the helical wiggler, but also contains a small component of Larmor procession due to the axial field.

\section{SPONTANEOUS EMISSION}

The time-averaged radiated power $P$ can be computed by means of the equation

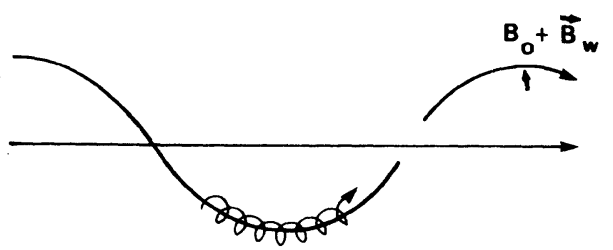

FIG. 7. Schematic representation of the singleparticle trajectories.

$$
P=-\lim _{T \rightarrow \infty} \frac{1}{T} \int_{-T / 2}^{T / 2} d t \int d^{3} x \overrightarrow{\mathrm{E}}(\overrightarrow{\mathrm{x}}, t) \cdot \overrightarrow{\mathrm{J}}(\overrightarrow{\mathrm{x}}, t)
$$

where $\overrightarrow{\mathrm{E}}(\overrightarrow{\mathrm{x}}, t)$ denotes the microscopic radiation field, and

$$
\overrightarrow{\mathrm{J}}(\overrightarrow{\mathrm{x}}, t)=-e \sum_{j=1}^{N_{b}} \overrightarrow{\mathrm{v}}_{j}(t) \delta\left(\overrightarrow{\mathrm{x}}-\overrightarrow{\mathrm{x}}_{j}(t)\right)
$$

is the source current composed of the sum of the microscopic currents due to all $N_{b}$ electrons in the beam during the interaction time $-T / 2<t<T / 2$. The radiated power can be expressed in terms of the Fourier amplitudes of the microscopic fields and source currents in the following manner:

$P=-2(2 \pi)^{4} \lim _{T \rightarrow \infty} \frac{1}{T} \int d^{3} k \int_{0}^{\infty} d \omega \operatorname{Re}\left(\overrightarrow{\mathrm{E}}_{\overrightarrow{\mathrm{k}}, \omega} \cdot \overrightarrow{\mathrm{J}}_{\overrightarrow{\mathrm{k}}, \omega}^{*}\right)$,

where the asterisk $\left(^{*}\right)$ denotes the complex conjugate, and the Fourier amplitude is defined as

$$
f_{\overrightarrow{\mathrm{k}}, \omega}=\int d^{3} k \int_{-\infty}^{\infty} d \omega \exp (i \omega t-i \overrightarrow{\mathrm{k}} \cdot \overrightarrow{\mathrm{x}}) f(\overrightarrow{\mathrm{x}}, t) .
$$

A self-consistent relation between the fields and source currents depends upon the dielectric properties of the beam, and can be written as

$$
\overleftrightarrow{\Lambda}_{\overrightarrow{\mathrm{k}}, \omega} \cdot \overrightarrow{\mathrm{E}}_{\overrightarrow{\mathrm{k}}, \omega}=\frac{4 \pi i}{\omega} \overrightarrow{\mathrm{J}}_{\overrightarrow{\mathrm{k}}, \omega},
$$

where the dispersion tensor

$$
\overleftrightarrow{\Lambda}_{\overrightarrow{\mathrm{k}}, \omega}=\left(1-n^{2}\right) \overleftrightarrow{I}+n^{2} \hat{k} \hat{k}+\overleftrightarrow{\epsilon}_{\overrightarrow{\mathrm{k}}, \omega},
$$

$n(\equiv c k / \omega)$ is the index of refraction, $\overleftrightarrow{I}$ is the unit dyadic $\hat{k} \equiv \overrightarrow{\mathbf{k}} /|\overrightarrow{\mathbf{k}}|$, and $\overleftrightarrow{\boldsymbol{\epsilon}}_{\overrightarrow{\mathrm{k}}, \omega}$ is the beam dielectric tensor. For the case of a diffuse beam, in which the radiation frequency greatly exceeds the electron plasma frequency, $\overleftrightarrow{\epsilon}_{\overrightarrow{\mathrm{k}}, \omega} \simeq \overleftrightarrow{I}$ and (12) can be inverted to give

$\overrightarrow{\mathrm{E}}_{\overrightarrow{\mathrm{k}}, \omega}=-\frac{(4 \pi i \omega)}{\left(\omega^{2}-c^{2} k^{2}\right)}\left[\overrightarrow{\mathrm{J}}_{\overrightarrow{\mathrm{k}}, \omega}-n^{2} \hat{k}\left(\hat{k} \cdot \overrightarrow{\mathrm{J}}_{\overrightarrow{\mathrm{k}}, \omega}\right)\right]$.

As a result, if the emissivity $\eta\left(\omega, \Omega_{\hat{k}}\right)$ is defined to be the power radiated per unit frequency and volume into the solid angle $\Omega_{\hat{k}}$ subtended by $\hat{k}$, then we find

$$
\begin{aligned}
\eta\left(\omega, \Omega_{\hat{k}}\right)= & (2 \pi)^{6}\left(\frac{\omega^{2}}{V c^{3}}\right) \\
& \times \lim _{T \rightarrow \infty} \frac{1}{T}\left(\left|J_{\vec{k}, \omega}\right|^{2}-\left|\hat{k} \cdot \overrightarrow{\mathbf{J}}_{\overrightarrow{\mathrm{k}}, \omega}\right|^{2}\right)_{k=\omega / c},
\end{aligned}
$$

where $V$ is the total volume of the interaction region. The radiation spectrum, therefore, can be 
determined from (15) with a knowledge of the single-particle orbits (7) necessary to compute the source current.

Using the trajectories in (7), we find that after transforming to the frame $\left(\hat{e}_{1}=\cos \psi \hat{e}_{x}+\sin \psi \hat{e}_{y}\right.$, $\hat{e}_{\psi}=-\sin \psi \hat{e}_{x}+\cos \psi \hat{e}_{y}$, and $\left.\tan \psi=k_{y} / k_{x}\right)$ in which

$$
\overrightarrow{\mathbf{k}}=k_{\perp} \hat{e}_{\perp}+k_{z} \hat{e}_{z}
$$

$\left[k_{\perp}=\left(k_{x}^{2}+k_{y}^{2}\right)^{1 / 2}\right]$, the source current is

$$
\begin{aligned}
\overrightarrow{\mathrm{J}}_{\overrightarrow{\mathbf{k}}, \omega}=-\frac{e}{(2 \pi)^{3}} \lim _{T \rightarrow \infty} \sum_{j=1}^{N_{b}} \exp \left(-i \overrightarrow{\mathrm{k}} \cdot \overrightarrow{\mathrm{x}}_{0}^{(j)}\right) \sum_{l, m, n=-\infty}^{\infty} & \overrightarrow{\mathrm{V}}_{l m n}^{(j)} \frac{\sin \left[\left(\omega-k_{z} v_{\|}^{(j)}-(l+n) k_{w} v_{\|}^{(j)}-(m-n) \Omega_{0}^{(j)}\right) T / 2\right]}{\pi\left[\omega-k_{z} v_{\|}^{(j)}-(l+n) k_{w} v_{\|}^{(j)}-(m-n) \Omega_{0}^{(j)}\right]} \\
& \times \exp \left[i b_{1}^{(j)} \sin \left(\phi^{(j)}-\psi\right)-i m\left(\phi^{(j)}-\psi\right)\right] \\
& \times \exp \left[i b^{(j)} \sin \left(k_{w} z_{0}^{(j)}-\psi\right)-i l\left(k_{w} z_{0}^{(j)}-\psi\right)\right] \\
& \times \exp \left[i b_{\|}^{(j)} \sin \left(k_{w} z_{0}^{(j)}-\phi^{(j)}\right)-i n\left(k_{w} z_{0}^{(j)}-\phi^{(j)}\right)\right],
\end{aligned}
$$

where $\overrightarrow{\mathrm{x}}_{0}^{(j)}$ defines the initial position of the $j$ th electron,

$$
\begin{aligned}
& \phi^{(j)} \equiv \tan ^{-1}\left(P_{y} / P_{x}\right), \quad b^{(j)} \equiv k_{\perp} \Omega_{w}^{(j)} / k_{w}\left(\Omega_{0}^{(j)}-k_{w} v_{\|}^{(j)}\right), \quad b_{\perp}^{(j)} \equiv k_{\perp} V_{\perp}^{(j)} / \Omega_{0}^{(j)}, \\
& b_{z}^{(j)} \equiv k_{z} V_{\perp}^{(j)} \Omega_{w}^{(j)} /\left(\Omega_{0}^{(j)}-k_{w} v_{\|}^{(j)}\right)^{2}, \text { and } V_{I}^{2} \equiv\left(P_{x}^{2}+P_{y}^{2}\right) / \gamma m .
\end{aligned}
$$

In addition,

$$
\begin{aligned}
\overrightarrow{\mathrm{V}}_{l m n}^{(j)}=J_{n}\left(b_{z}^{(j)}\right) & {\left[\left[\frac{l k_{w} v^{(j)}+m \Omega_{0}^{(j)}}{k_{\perp}}\right] J_{l}\left(b^{(j)}\right) J_{m}\left(b_{\perp}^{(j)}\right) \hat{e}_{\perp}+i\left[v_{w}^{(j)} J_{l}^{\prime}\left(b^{(j)}\right) J_{m}\left(b_{\perp}^{(j)}\right)+V_{\perp}^{(j)} J_{l}\left(b^{(j)}\right) J_{m}^{\prime}\left(b_{\perp}^{(j)}\right)\right] \hat{e}_{\psi}\right.} \\
& \left.+\left[v_{\|}^{(j)}-\frac{n\left(\Omega_{0}^{(j)}-k_{w} v_{\|}^{(j)}\right)}{k_{\perp}}\right] J_{l}\left(b^{(j)}\right) J_{m}\left(b_{\perp}^{(j)}\right) \hat{e}_{z}\right]
\end{aligned}
$$

where $v_{w} \equiv \Omega_{w} v_{\|} /\left(\Omega_{0}-k_{w} v_{\|}\right)$, and $J_{p}$ and $J_{p}^{\prime}$ are the regular Bessel function of the first kind and its derivative, respectively. In computing the quadratic forms of the source current which appear in the emissivity (15), we impose a random-phase approximation to obtain

$$
\begin{array}{r}
\eta\left(\omega, \Omega_{\hat{k}}\right)=\frac{e^{2} \omega^{2}}{2 \pi c^{3} V} \sum_{j=1}^{N_{b}} \sum_{l, m, n=-\infty}^{\infty} \sum_{l^{\prime}, m^{\prime}, n^{\prime}=-\infty}^{\infty} \delta_{l+n, l^{\prime}+n^{\prime}} \delta_{m-n, m^{\prime}-n^{\prime}}\left(\overrightarrow{\mathrm{V}}_{l m n}^{(j)} \cdot \overrightarrow{\mathrm{V}}_{l^{\prime} m^{\prime} n^{\prime}}^{(j)^{*}}-\hat{k} \cdot \overrightarrow{\mathrm{V}}_{l m n}^{(j)} \hat{k^{\prime} \cdot \overrightarrow{\mathrm{V}}_{l^{\prime} m^{\prime} n^{\prime}}^{(j)}}\right) \\
\times \delta\left(\omega-k_{z} v_{l l^{*}}^{(j)}-(l+n) k_{w} v_{l \mid}^{(j)}-(m-n) \Omega_{0}^{(j)}\right)_{k=\omega / c} .
\end{array}
$$

We treat the emissivity in the limit in which $b_{z}^{(j)}<<1$, which is generally valid as long as $2 \gamma_{z}^{2} \in \beta_{0}\left(V_{\perp} / v_{\|}\right)<<1$. When this condition is satisfied the dominant terms in (18) are those for which $n=n^{\prime}=0$, and the emissivity takes on the relatively simple form

$$
\begin{aligned}
\eta\left(\omega, \Omega_{\hat{k}}\right) \simeq \frac{e^{2} \omega^{2}}{2 \pi c^{3} V} \sum_{j=1}^{N_{b}} \sum_{l, m=-\infty}^{\infty} & \left\{\left[\mid \frac{l k_{w} v_{l}^{(j)}+m \Omega_{0}^{(j)}}{k_{\perp}}\right] \cos \theta-v_{\|}^{(j)} \sin \theta\right]^{2} J_{l}^{2}\left(b^{(j)}\right) J_{m}^{2}\left(b_{\perp}^{(j)}\right) \\
+ & {\left.\left[v_{w}^{(j)} J_{l}^{\prime}\left(b^{(j)}\right) J_{m}\left(b_{l}^{(j)}\right)+V_{1}^{(j)} J_{l}\left(b^{(j)}\right) J_{m}^{\prime}\left(b_{I}^{(j)}\right)\right]^{2}\right\} } \\
& \times \delta\left(\omega-k_{z} v_{l}^{(j)}-l k_{w} v_{l}^{(j)}-m \Omega_{0}^{(j)}\right)_{k=\omega / c},
\end{aligned}
$$

where $\theta \equiv \cos ^{-1}\left(\hat{k} \cdot \hat{e}_{z}\right)$ is the polar angle between the wave vector and the axial guide field.

We now convert the discrete sum over individual electrons in (18) and (19), into a continuous integral 
over the beam distribution function by making the replacement $V^{-1} \sum_{j=1}^{N_{b}} \rightarrow n_{b} \int d P_{x} d P_{y} d p F_{b}$, where $n_{b} \equiv N_{b} / V$, and the beam distribution $F_{b}$ is assumed to be a function of $\left(P_{x}, P_{y}, p\right)$. As a consequence

$$
\begin{aligned}
\eta\left(\omega, \Omega_{\hat{k}}\right) \simeq \frac{e^{2} n_{b} \omega^{2}}{2 \pi c^{3}} \int d P_{x} d P_{y} d p F_{b} \sum_{l, m=-\infty}\{ & \left\{\left[\frac{l k_{w} v_{\|}+m \Omega_{0}}{k_{\perp}}\right] \cos -v_{\|} \sin \theta\right]^{2} J_{l}^{2}(b) J_{m}^{2}\left(b_{\perp}\right) \\
+ & {\left.\left[v_{w} J_{l}^{\prime}(b) J_{m}\left(b_{\perp}\right)+V_{\perp} J_{l}(b) J_{m}^{\prime}\left(b_{\perp}\right)\right]^{2}\right\} } \\
& \times \delta\left(\omega-k_{z} v_{\|}-l k_{w} v_{\|}-m \Omega_{0}\right)_{k=\omega / c},
\end{aligned}
$$

where $v_{\|}$is determined by means of Eq. (8). Since (8) is, in general, a quartic polynomial for $v_{\|}$, care must be exercised in selecting the appropriate root. In the limit of propagation parallel to the axial guide field, (20) reduces to the comparatively simple form

$$
\eta\left(\omega, \Omega_{\hat{k}=\hat{e}_{z}}\right) \simeq \frac{e^{2} n_{b} \omega^{2}}{2 \pi c} \int d P_{x} d P_{y} d p F_{b}\left[\frac{v_{w}^{2}}{c^{2}} \delta\left[\omega-\left(k+k_{w}\right) v_{\|}\right]+\frac{V_{1}^{2}}{c^{2}} \delta\left(\omega-k v_{\|}-\Omega_{0}\right)\right]_{k=\omega / c} .
$$

Thus, the incoherent radiation corresponding to the usual free-electron-laser resonance at $\omega \sim 2 \gamma_{z}^{2} k_{w} v_{\|}$scales as $v_{w}^{2}$. The emission at the cyclotron resonance is expected to be much less intense in this regime in which it is required that $V_{\perp}^{2}<v_{w}^{2}$ in the derivation of the relativistic trajectories (7).

In order to illustrate typical noise levels and spectra to be expected in the course of operation of a freeelectron laser we assume a distribution of the form $F\left(P_{x}, P_{y}, p\right)=n_{b} \delta\left(P_{x}\right) \delta\left(P_{y}\right) G_{b}(p)$, and model the variation in total momentum with a distribution of the form

$$
G_{b}(p)=\frac{\Delta p}{\pi} \frac{1}{\left(p-p_{0}\right)^{2}+\Delta p^{2}},
$$

where $p_{0}$ characterizes the bulk momentum in the beam and $\Delta p$ describes the momentum spread. Note that since $P_{x}=P_{y}=0$, the component of the motion describing Larmor rotation is assumed to be negligible, and the noise spectrum is predominantly associated with the free-electron-laser resonance. As a consequence, the emissivity is of the form

$$
\eta\left(\omega, \Omega_{\hat{k}=\hat{z}_{2}}\right) \simeq \frac{\Omega_{w}^{2}}{8 \pi^{3} k_{w}^{2}} \frac{m \omega_{b}^{2}}{c^{3}} \frac{\omega^{4}}{\left(\omega+k_{w} c\right)^{3}} \frac{\left(1+u_{\mathrm{ph}}^{2}\right)^{3 / 2}}{\left(\beta_{0} \gamma_{0}-u_{\mathrm{ph}}\right)^{2}} \frac{\Delta u}{\left(u_{\mathrm{ph}}-u_{0}\right)^{2}+\Delta u^{2}},
$$

where $\omega_{b}^{2} \equiv 4 \pi e^{2} n_{b} / m, \Delta u \equiv \Delta p / m c, u_{0} \equiv p_{0} / m c$, and

$$
u_{\mathrm{ph}}=\frac{\omega}{k_{w} c}\left(\frac{1+\Omega_{w}^{2} / k_{w}^{2} c^{2}}{1+2 \omega / k_{w} c}\right)^{1 / 2} \text {. }
$$

As an example, we shall evaluate the emissivity (22) for a 1-A electron beam characterized by a bulk energy of $1.4 \mathrm{MeV}$ with a $3 \%$ energy spread and a $3-\mathrm{cm}$ beam radius. The beam density, therefore, is approximately $n_{b} \simeq 1 \times 10^{9} \mathrm{~cm}^{-3}$. In addition, we shall assume the wiggler amplitude and period to be $700 \mathrm{G}$ and 3 $\mathrm{cm}$, respectively, and the axial guide field to be $15 \mathrm{kG}$. The resulting noise spectrum is shown in Fig. 8 . The peak intensity is of the order of $0.013 \mathrm{pW} \mathrm{Hz}^{-1} \mathrm{~cm}^{-3}$ and occurs at a frequency of approximately 240 $\mathrm{GHz}$ with a half-width of $6 \%$.

\section{COHERENT EMISSION}

In this section we derive the linearized gain by solution of the Vlasov-Maxwell equations. If the distribution is written as the sum of equilibrium and fluctuating components $f_{b}(z, \overrightarrow{\mathrm{p}}, t)=F_{b}\left(P_{x}, P_{y}, p\right)$ $+\delta f_{b}(z, \overrightarrow{\mathrm{p}}, t)$, then the formal solution of the Vlasov equation for the perturbed distribution is 


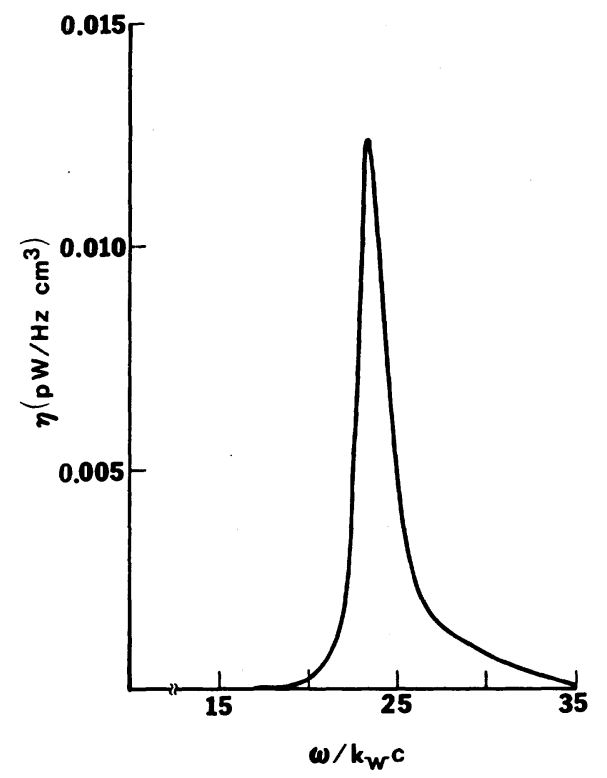

FIG. 8. Graph of the emissivity versus frequency for propagation parallel to the axial guide field.

$$
\begin{aligned}
\delta f_{b}(z, \overrightarrow{\mathrm{p}}, t(z))= & e \int_{0}^{z} \frac{d z^{\prime}}{v_{z}\left(z^{\prime}\right)} \delta \overrightarrow{\mathrm{E}}\left(z^{\prime}, t\left(z^{\prime}\right)\right. \\
& +\frac{1}{c} \overrightarrow{\mathrm{v}}\left(z^{\prime}\right) \times \delta \overrightarrow{\mathrm{B}}\left(z^{\prime}, t\left(z^{\prime}\right)\right) \cdot \frac{\partial F_{b}}{\partial \overrightarrow{\mathrm{p}}\left(z^{\prime}\right)}
\end{aligned}
$$

to first order in the radiation fields, where the solution is parametrized in terms of the axial distance from the start of the interaction region (at $z=0$ ), and $t(z) \equiv t_{0}+\int_{0}^{z} d z^{\prime} / v_{z}\left(z^{\prime}\right)$ is the sum of the time required for an electron to traverse the distance and the entry time $t_{0}$. The transverse components of the trajectory, therefore, are written in the form $p_{x}=p_{w} \cos k_{w} z+P_{x} \cos \Omega_{0} t(z)-P_{y} \sin \Omega_{0} t(z)$, and $p_{y}=p_{w} \sin k_{w} z+P_{x} \sin \Omega_{0} t(z)+P_{y} \cos \Omega_{0} t(z)$.

We assume plane-wave solutions of the form $\exp (i \omega t)$ and choose to work with scalar and vector potentials of the form

$$
\delta \phi(z, t)=\frac{1}{2} \delta \hat{\phi}(z) \exp (-i \omega t)+\text { c.c. }
$$

and

$$
\delta \overrightarrow{\mathrm{A}}(z, t)=\frac{1}{2} \delta \hat{A}(z) \exp (-i \omega t)+c . c .
$$

where it is evident that $\hat{e}_{\mathbf{z}} \cdot \delta \overrightarrow{\mathrm{A}}=0$. After transformation to the basis $\hat{e}_{ \pm}=\frac{1}{2}\left(\hat{e}_{x} \pm i \hat{e}_{y}\right)$, therefore, the perturbed distribution can be written as $\delta f_{b}(z, \overrightarrow{\mathrm{p}}, \tau(z))=\delta \widehat{f}_{b}(z, \overrightarrow{\mathrm{p}}) \exp [-i \omega t(z)]+$ c.c.,

where

$$
\begin{aligned}
\delta \hat{f}_{b}(z, \overrightarrow{\mathrm{p}})=\frac{e}{2 c} \int_{0}^{z} d z^{\prime} \frac{\exp \left[i \omega \tau\left(z, z^{\prime}\right)\right]}{v_{z}\left(z^{\prime}\right)}\{ & \left\{-c p_{z}\left(z^{\prime}\right) \partial_{z^{\prime}} \delta \hat{\phi}\left(z^{\prime}\right)+i \omega\left(p_{-}\left(z^{\prime}\right) \delta \hat{A}_{+}\left(z^{\prime}\right)+p_{+}\left(z^{\prime}\right) \delta \hat{A}_{-}\left(z^{\prime}\right)\right]\right\} \frac{1}{p} \frac{\partial}{\partial p} \\
& +\exp \left[i \Omega_{0} t\left(z^{\prime}\right)\right]\left[i \omega-v_{z}\left(z^{\prime}\right) \partial_{z^{\prime}}\right] \delta \hat{A}_{+}\left(z^{\prime}\right)\left(\frac{\partial}{\partial P_{x}}+i \frac{\partial}{\partial P_{y}}\right) \\
& \left.+\exp \left[-i \Omega_{0} t\left(z^{\prime}\right)\right]\left[i \omega-v_{z}\left(z^{\prime}\right) \partial_{z^{\prime}}\right] \delta \hat{A}_{-}\left(z^{\prime}\right)\left(\frac{\partial}{\partial P_{x}}-i \frac{\partial}{\partial P_{y}}\right)\right\} F_{b}\left(P_{x}, P_{y}, p\right),
\end{aligned}
$$

$p_{ \pm} \equiv p_{x} \mp i p_{y}, \quad \delta \hat{A}_{ \pm} \equiv \frac{1}{2}\left(\delta \hat{A}_{x} \mp i \delta \hat{A}_{y}\right)$, and $\tau\left(z, z^{\prime}\right) \equiv \int_{z^{\prime}}^{z} d z^{\prime \prime} / v_{z}\left(z^{\prime \prime}\right)$.

Using (24) the perturbed current,

$$
\delta \vec{J}(z, t)=\left[\delta \hat{J}_{+}(z) \hat{e}_{+}+\delta J_{-}(z) \hat{e}_{-}+\delta \hat{J}_{z}(z) \hat{e}_{z}\right] \exp (-i \omega t)+c . c .,
$$

can be computed as follows:

$$
\left(\begin{array}{c}
\delta \hat{J}_{ \pm} \\
\delta \hat{J}_{z}
\end{array}\right)=-\frac{e}{m} \int d P_{x} d P_{y} d p \frac{p}{\gamma p_{z}} \delta \hat{f}_{b}(z, \overrightarrow{\mathrm{p}})\left(\begin{array}{c}
p_{ \pm} \\
p_{z}
\end{array}\right]
$$

Since the assumption of small $P_{x}$ and $P_{y}$ is central to the analysis, we shall adopt an equilibrium distribution of the form

$$
F_{b}\left(P_{x}, P_{y}, p\right)=n_{b} \delta\left(P_{x}\right) \delta\left(P_{y}\right) G_{b}(p),
$$

in the interest of computational simplicity. Here $G_{b}(p)$ is an arbitrary function of the magnitude of the momentum subject only to the normalization $\int_{0}^{\infty} d p p G_{b}(p) / p_{z}=1$. Using (26), we find the perturbed 
currents

$$
\begin{aligned}
& \delta \hat{J}_{ \pm}=\frac{\omega_{b}^{2}}{8 \pi c} \int_{0}^{\infty} d p \frac{p}{p_{z} \gamma} \exp \left[\mp i \Omega_{0} t(z)\right]\left(2+\frac{p_{+} p_{-}}{p_{z}^{2}}\right) D_{ \pm}+\exp \left[ \pm i \Omega_{0} t(z)\right] \frac{p_{ \pm}^{2}}{p_{z}^{2}} D_{\mp} \\
&\left.+p_{ \pm}\left(\frac{\partial}{\partial P_{x}}+i \frac{\partial}{\partial P_{y}}\right) D_{+}+p_{ \pm}\left(\frac{\partial}{\partial_{x}}-i \frac{\partial}{\partial P_{y}}\right) D_{-}-p_{ \pm} D_{z} \frac{\partial}{\partial P}\right]_{P_{x}=P_{y}=0} G_{b}(p) \\
& \delta \hat{J}_{z}=\frac{\omega_{b}^{2}}{8 \pi c} \int_{0}^{\infty} d p \frac{p}{\gamma}\left[\left(\frac{\partial}{\partial P_{x}}+i \frac{\partial}{\partial P_{y}}\right) D_{+}+\left(\frac{\partial}{\partial P_{x}}-i \frac{\partial}{\partial P_{y}}\right) D_{-}-D_{z} \frac{\partial}{\partial p}\right]_{P_{x}=P_{y}=0} G_{b}(p)
\end{aligned}
$$

where

$$
\begin{aligned}
& p_{z}=p_{\|}\left[\equiv\left(p^{2}-p_{w}^{2}\right)^{1 / 2}\right], \quad p_{ \pm}=p_{w} \exp \left(\mp i k_{w} z\right), \quad \tau\left(z, z^{\prime}\right)=\left(z-z^{\prime}\right) / v_{\|}, \omega_{b}^{2} \equiv 4 \pi e^{2} n_{b} / m, \\
& D_{ \pm} \equiv-\exp \left[ \pm i \Omega_{0} t(z)\right]\left(\delta \hat{A}_{ \pm}(z)-\delta \hat{A}_{ \pm}(0) \exp \left[i\left(\omega \mp \Omega_{0}\right) z / v_{z}\right] \mp i \frac{\Omega_{0}}{v_{z}} \int_{0}^{z} d z^{\prime} \delta \hat{A}_{ \pm}\left(z^{\prime}\right) \exp \left[i\left(\omega \pm \Omega_{0}\right) \tau\left(z, z^{\prime}\right)\right]\right),
\end{aligned}
$$

and

$$
D_{z} \equiv \frac{\gamma m c}{p} \int_{0}^{z} d z^{\prime} \exp \left[i \omega \tau\left(z, z^{\prime}\right)\right]\left[-\partial_{z^{\prime}} \delta \hat{\phi}\left(z^{\prime}\right)+\frac{i \omega}{c}\left(\frac{p_{-}}{p_{z}} \delta \hat{A}_{+}\left(z^{\prime}\right)+\frac{p_{+}}{p_{z}} \delta \hat{A}_{-}\left(z^{\prime}\right)\right]\right]
$$

for $P_{x}=P_{y}=0$. The dispersion equations are obtained by using these perturbed currents in the wave equations:

$$
\begin{aligned}
& \left(\partial_{z}^{2}+\frac{\omega^{2}}{c^{2}}\right) \delta \hat{A}_{ \pm}(z)=-\frac{4 \pi}{c} \delta \hat{J}_{ \pm}, \\
& \partial_{z} \delta \hat{\phi}(z)=\frac{8 \pi i}{\omega} \delta \hat{J}_{z} .
\end{aligned}
$$

We are primarily interested in the low-gain, tenuous-beam limit in which $\omega_{b}<<\omega$ and the wave amplitudes vary little over the length of the interaction region. This is the regime appropriate to the experiments conducted at Stanford University. ${ }^{1,2}$ In this regime collective effects are unimportant and the space-charge potential can be neglect- ed. In addition, the coupling between the electromagnetic modes described by $\delta \hat{A}_{+}$and $\delta \hat{A}_{-}$ scales with $p_{w}^{2} / p_{z}^{2}$. However, the assumption of low gain implies that this parameter is small, and we can focus attention on one or the other of these modes exclusively. We choose to consider $\delta \hat{A}_{+}$, which in the low-gain regime can be represented as

$$
\delta \hat{A}_{+}(z)=\delta \hat{A}_{+}(0) \exp \left[i \int_{0}^{z} d z^{\prime} k_{+}\left(z^{\prime}\right)\right],
$$

where $\left|\operatorname{Im} k_{+}(z)\right|<<\left|\operatorname{Re} k_{+}(z)\right|$. Under the assumption that $k_{+}(z)=k+\delta k_{+}(z)$, where $k$ is independent of axial position and $\left|\delta k_{+}(z) / k\right|<<1$, the dispersion equation becomes

$$
k^{2}-\omega^{2} / c^{2} \simeq 0,
$$

and

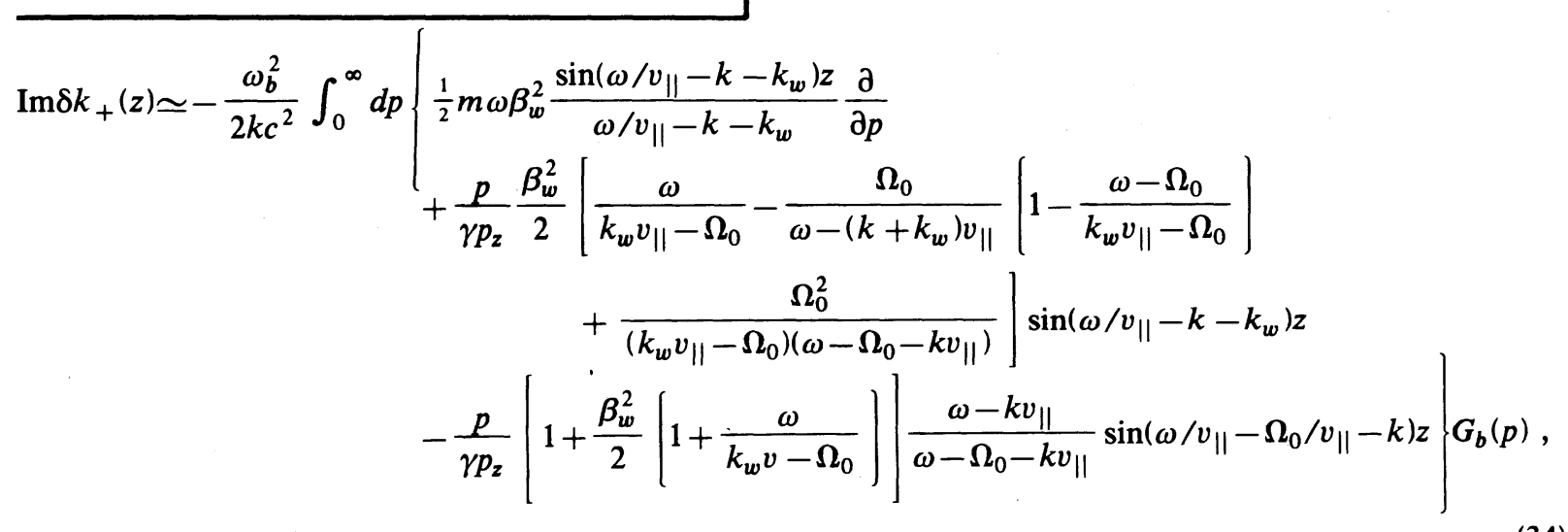


where $\beta_{w}^{2} \equiv p_{w}^{2} / p_{z}^{2}$. This result reduces to that found by Sprangle and $\mathrm{Smith}^{4}$ in the limit of a vanishing axial magnetic field.

The total gain over an interaction region of length $L$ is defined as $G_{L} \equiv-\int_{0}^{L} d z \operatorname{Im} \delta k_{+}(z)$. Integrating (34), therefore, we find

$$
\begin{aligned}
G_{L} \simeq-\frac{\omega_{b}^{2} L}{2 k c^{2}} \int_{0}^{\infty} d p G_{b}(p)\{ & \left.\left.\frac{m \omega L}{4} \frac{\partial}{\partial p}\left[\beta_{w}^{2} \frac{\sin ^{2} \theta_{w}}{\theta_{w}^{2}}\right)-\frac{p}{\gamma p_{z}} \frac{\beta_{w}^{2}}{2} \frac{\omega}{k_{w} v_{\|}-\Omega_{0}}\right] 1+\frac{\Omega_{0} L}{2 v_{\|}} \frac{\Omega_{0}}{\omega \theta_{g}}\right) \frac{\sin ^{2} \theta_{w}}{\theta_{w}} \\
& +\frac{p}{\gamma p_{z}} \frac{\beta_{w}^{2}}{2} \frac{\Omega_{0} L}{2 v_{\|}}\left[1-\frac{\omega-\Omega_{0}}{k_{w} v_{\|}-\Omega_{0}}\right) \frac{\sin ^{2} \theta_{w}}{\theta_{w}^{2}} \\
& \left.-\frac{p}{\gamma p_{z}}\left[1+\frac{\beta_{w}^{2}}{2}\left[1+\frac{\omega}{k_{w} v_{\|}-\Omega_{0}}\right]\right]\left[\theta_{g}+\frac{\Omega_{0} L}{2 v_{\|}}\right] \frac{\sin ^{2} \theta_{g}}{\theta_{g}^{2}}\right\},
\end{aligned}
$$

where $\theta_{w} \equiv\left(\omega / v_{\|}-k-k_{w}\right) L / 2$ and $\theta_{g} \equiv\left(\omega / v_{\|}-\Omega_{0} / v_{\|}-k\right) L / 2$. If the beam is sufficiently cold that a distribution of the form

$$
G_{b}(p)=\frac{p_{z}}{p} \delta\left(p-p_{0}\right)
$$

can be used, then the total gain becomes

$$
\begin{aligned}
G_{L} \simeq \frac{\omega_{b}^{2}}{2 k c^{2}} \frac{L}{\gamma_{0}}\left(\frac { \beta _ { w 0 } ^ { 2 } } { 2 } \left\{\frac{\omega^{2} L^{2}}{4 \gamma_{z 0}^{2} v_{\| 0}^{2}}\left[1-\frac{\gamma_{z 0}^{2} \beta_{w 0}^{2} \Omega_{0}}{\Omega_{0}\left(1+\beta_{w 0}^{2}\right)-k_{w} v_{\| 0}}\right) \frac{\partial}{\partial \theta_{w 0}}\left(\frac{\sin \theta_{w 0}}{\theta_{w 0}}\right)^{2}\right.\right. \\
-k_{w} L\left[\frac{\omega}{\Omega_{0}\left(1+\beta_{w 0}^{2}\right)-k_{w} v_{\| 0}}+\frac{\Omega_{0}}{2 k_{w} v_{\| 0}}\left[1-\frac{\omega-\Omega_{0}}{k_{w} v_{\| 0}-\Omega_{0}}\right)\right]\left[\frac{\sin \Omega_{w 0}}{\theta_{w 0}}\right]^{2} \\
\left.+\frac{\omega}{k_{w} v_{\| 0}-\Omega_{0}}\left[1+\frac{\Omega_{0}}{\omega} \frac{\Omega_{0} L}{2 v_{\| 0}} \frac{1}{\theta_{g 0}}\right) \frac{\sin ^{2} \theta_{w 0}}{\theta_{w 0}}\right\} \\
\left.-\left[1+\frac{\beta_{w 0}^{2}}{2}\left[1+\frac{\omega}{k_{w} v_{\| 0}-\Omega_{0}}\right]\right]\left(1+\frac{\Omega_{0} L}{2 v_{\| 0}} \frac{1}{\theta_{g 0}}\right) \frac{\sin ^{2} \theta_{g 0}}{\theta_{g 0}}\right)
\end{aligned}
$$

In Eq. (37),

$$
\begin{aligned}
& \gamma_{0} \equiv\left(1+p_{0}^{2} / m^{2} c^{2}\right)^{1 / 2}, \gamma_{z 0} \equiv\left(1-v_{\| 0}^{2} / c^{2}\right)^{-1 / 2}, \beta_{w 0}^{2} \equiv p_{w 0}^{2} / p_{\| 0}^{2}, \\
& p_{w 0}^{2} \equiv \Omega_{w}^{2} p_{\| 0}^{2} /\left(\Omega_{0}-k_{w} v_{\| 0}\right)^{2},
\end{aligned}
$$

and

$$
p_{\| 0} \text { and } v_{\| 0}\left(\equiv p_{\| 0} / \gamma_{0} m\right)
$$

are determined by the appropriate solution to $p_{0}^{2}=p_{w 0}^{2}+p_{\| 0}^{2}$. It is clear from (37), therefore, that wave amplification can occur, in principle, for frequencies corresponding to both the usual free-electron-laser resonance $\left[\omega \simeq\left(k+k_{w}\right) v_{\| 0}\right]$ and the Doppler-shifted gyroresonance $\left(\omega \simeq \Omega_{0}+k v_{\| 0}\right)$.

If the pump period is short compared to the length of the system, the gain for frequencies $\omega \simeq\left(k+k_{w}\right) v_{\| 0}$ is dominated by first term in (37),

$$
G_{L} \simeq \beta_{w 0}^{2} \frac{\omega_{b}^{2} L^{3} k}{16 \gamma_{0} \gamma_{z 0}^{2} v_{\| 0}^{2}}\left(1-\frac{\gamma_{z 0}^{2} \beta_{w 0}^{2} \Omega_{0}}{\Omega_{0}\left(1+\beta_{w 0}^{2}\right)-k_{w} v_{\| 0}}\right) \frac{\partial}{\partial \theta_{w 0}}\left(\frac{\sin \theta_{w 0}}{\theta_{w 0}}\right)^{2} .
$$

The extrema occur for $\theta_{w 0} \simeq \pm 1.3$ at which $\partial\left(\sin \theta_{w 0} / \theta_{w 0}\right)^{2} / \partial \theta_{w 0} \simeq \mp 0.54$, which correspond to frequencies 


$$
\omega \simeq 2 \gamma_{z 0}^{2} k_{w} v_{\| 0}\left(1 \pm 2.6 / k_{w} L\right),
$$

and peak gains

$$
\left(G_{L}\right)_{\max } \simeq \pm 0.068 \beta_{w 0}^{2} \frac{\omega_{b}^{2}}{\gamma_{0} k_{w}^{2} c^{2}}\left(k_{w} L\right)^{3}\left(1-\frac{\gamma_{z 0}^{2} \beta_{w 0}^{2} \Omega_{0}}{\Omega_{0}\left(1+\beta_{w 0}^{2}\right)-k_{w} v_{\| 0}}\right),
$$

where the plus or minus sign must be chosen to give a positive gain in (40). These expressions for the gain are similar to those found previous$1 y^{4,19-22}$ in the limit of zero axial field. The principal differences between this result and those found previously are that (1) gain can occur for both frequencies shown in (39), and (2) a singularity is introduced for $k_{w} v_{\| 0} \simeq \Omega_{0}\left(1+\beta_{w 0}^{2}\right)$, which implies that the effect of the axial guide field can be to substantially enhance the small-signal gain over the case in which no guide field is present. Note, however, that the singularity is a product of our choice of a monoenergetic beam. The inclusion of an energy spread can be expected to broaden the resonance and remove the singularity.

We now focus on the effect of the axial magnetic field on the gain for the free-electron-laser resonance. If $\Delta$ denotes the ratio of the gain in the presence of an axial guide field to the appropriate expression in the limit $B_{0} \rightarrow 0$, then it follows that

$$
\Delta=-\mu^{2} \frac{\epsilon^{2} \beta_{v}^{2}+(\mu-1)\left[\epsilon^{2}+(\mu-1)^{2} \gamma_{0}^{-2}\right]}{\left[\epsilon^{2}-(\mu-1)^{3}\right]\left[\epsilon^{2}+(\mu-1)^{2} \gamma_{0}^{-2}\right]},
$$

where $\mu \equiv k_{w} v_{\| 0} / \Omega_{0}$ and $v_{\| 0}$ must be computed in a self-consistent manner to recover the constant $-v_{3}$ solutions in (5). As a result, $\Delta$ can be expressed as a function of $\epsilon, \beta_{v}$, and $\beta_{0}$ (where the choice of constant $-v_{3}$ solutions implicitly selects $\beta_{u}$ ). With these considerations in mind we plot $\Delta$ vs $\beta_{0}$ for fixed values of total electron energy and wiggler period and amplitude in Fig. 9(c). We shall restrict consideration to stable uniform- $v_{3}$ trajectories propagating parallel to the axial guide field. We choose $\beta_{v}=0.97$ and $\Omega_{w} / k_{w} c=0.07$ and plot the variation in $v_{\| 0}$ with $\beta_{0}$ in Fig. 9(a). Evidently there is a stable high axial velocity trajectory (orbit I) in the low- $\beta_{0}$ regime ( $\leqslant 0.72$ for the chosen parameters), as well as a trajectory which varies widely in magnitude with $\beta_{0}$ (orbit II). Since the frequency of the amplified modes scale approximately as $v_{|| 0}$, an electron beam characterized by trajectories in group I can be expected to excite high-frequency waves for all accessible values of $\beta_{0}$. In contrast, trajectories in group II can give rise to a wide spectrum of oscillations and, for the chosen parameters, and high-frequency waves will result only for $\beta_{0} \gtrsim 1$. This is clearly shown in Fig. 9(b) in which we plot the resonant frequency versus $\beta_{0}$ for both classes of orbits. The significant feature which we are concerned with, however, is the effect of the axial guide field on the small-signal gain. To this end, we plot $\Delta$ vs $\beta_{0}$ in Fig. 9(c) for the stated parameters. We observe, first, that $\Delta \geq 1$ throughout the entire range of $\beta_{0}$ accessible to orbits in group I and, hence, the gain is enhanced relative to the zero axial field $\left(\beta_{0} \rightarrow 0\right)$ limit. In addition, significant enhancements in the gain are possible near the indicated resonance at

$$
\beta_{0}=\left(1+\epsilon^{2 / 3}\right)^{-1} v_{\| 0} / c,
$$

which is the orbital stability boundary discussed previously in the regime in which $\beta_{0}<v_{\| 0} / c$. The resonance condition is not found for orbits in group II, however, since $\beta_{0}>v_{\| 0} / c$ for all such trajectories. Indeed, $\Delta$ vanishes for $\beta_{0} \simeq 1.39$ in the range of axial fields studied. Enhancement in the gain for these orbits occur only for limited ranges of $\beta_{0}$. A significant enhancement is found in the low- $\beta_{0}$ regime (i.e., $0.1 \leq \beta_{0} \leq 1.34$ ), but corresponds to comparatively low-frequency waves [see Fig. 9(b)]. Enhancements in the gain for highfrequency waves is found only for a limited range of axial field strengths $\left(1.45 \leqq \beta_{0} \lesssim 1.8\right)$, but is at most of the order of $35 \%$.

The enhancement in the gain for axial fields such that $\beta_{0}$ is of the order of unity is readily explained from consideration of the behavior of $v_{w}$. It is clear that for axial fields in which $\Omega_{0} \sim k_{w} v_{\| 0}$ (i.e., $\beta_{0} \sim 1$ ) large enhancements in the wiggle velocity $v_{w}$ occur. This is the source in the enhancements in the gain. However, as $\overrightarrow{\mathbf{B}}_{0}$ continues to increase $v_{w} \rightarrow \epsilon v_{\| 0}$, and the wiggle velocity decreases relative to that found in the zero axial field limit.

In the absence of an axial guide field the smallsignal gain scales as the square of the wiggler amplitude (i.e., $\Omega_{w}^{2} / k_{w}^{2} c^{2}$ ) which is also the square of the wiggle velocity. However, for finite values of the axial guide field this variation is more complex and the gain does not increase monotonically with either $\Omega_{w} / k_{w} c$ or the wiggle velocity. This situation is illustrated in Fig. 10, in which we plot the 


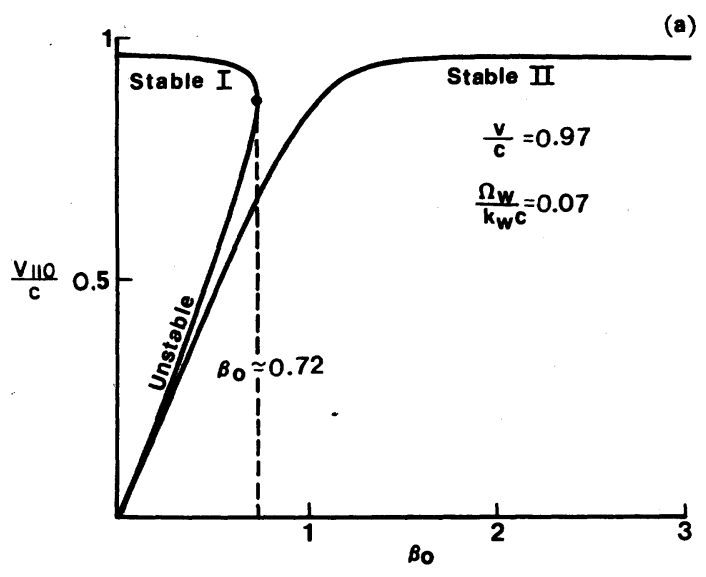

(b)
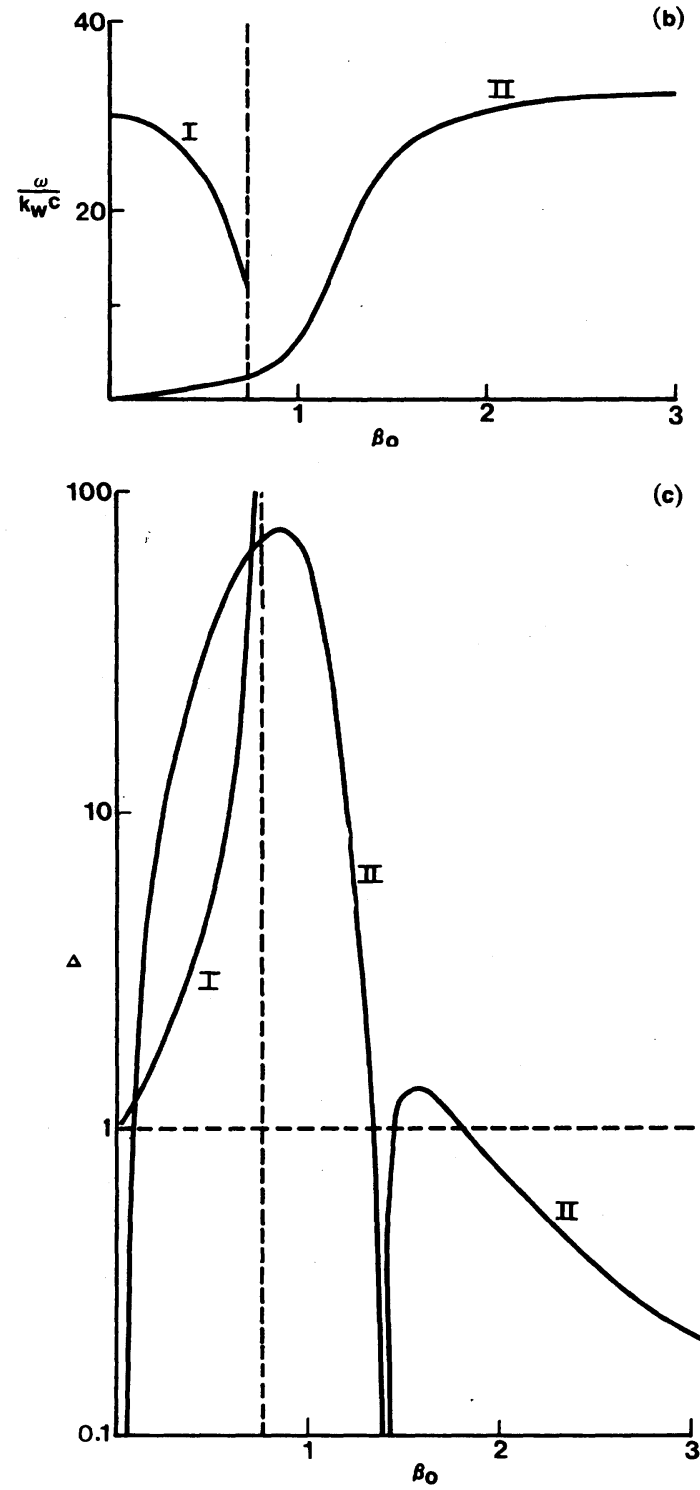

FIG. 9. Graphs of axial velocity, and resonant frequencies and enhancements in the gain for stable trajectories versus $\beta_{0}$ for $v / c=0.97$ and $\Omega_{w} / k_{w} c=0.07$.

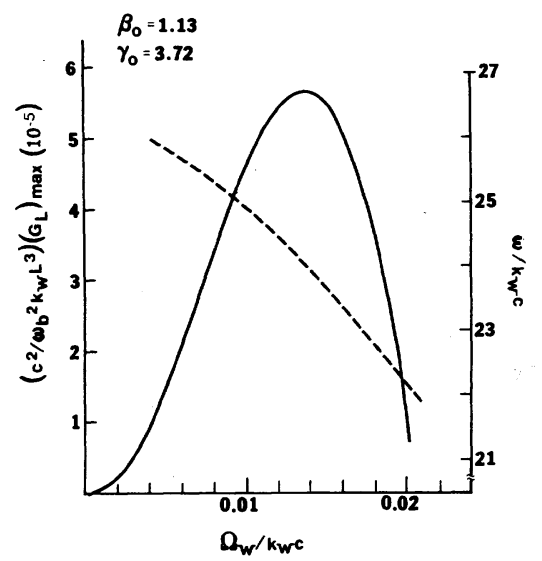

FIG. 10. Graphs of the maximum gain (solid line) and the corresponding frequency (dashed line) versus wiggler strength for $\beta_{0}=1.13$ and $v / c=0.96$.

peak gain (solid line) and frequency corresponding to peak gain (dashed line) versus $\Omega_{w} / k_{w} c$ for $\beta_{0}=1.13$ and $v / c=0.96$. For small values of this parameter the gain does indeed scale as the square of the wiggler amplitude, but a peak occurs at $\Omega_{w} / k_{w} c \simeq 0.012$ after which the gain rapidly drops to zero. In addition, the frequency monotonically decreases throughout. Note that while $\Omega_{w} / k_{w} c$ does not equal the wiggle velocity in the presence of an axial field, the decreasing frequency implies a decreasing axial velocity which, in turn, leads to an increasing wiggle velocity. This is illustrated more clearly in Fig. 11 in which we plot the variation in the axial velocity versus $\Omega_{w} / k_{w} c$ for $v / c=0.96$ and $\beta_{0}=0.75$ and 1.13. For $\beta_{0}=1.13$ all orbits are of the group-II class and the axial velocity is a decreasing function of $\Omega_{w} / k_{w} c$ over the range studied. Since this means that the wiggle velocity increases with the wiggler amplitude over this range,

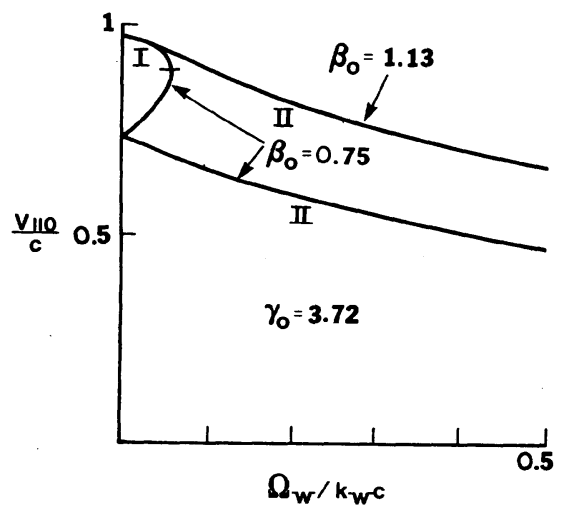

FIG. 11. Graph of the axial velocity versus $\Omega_{w} / k_{w} c$ for $v / c=0.96$, and $\beta_{0}=0.75$ and 1.13 . 
it must be concluded that the small-signal gain cannot necessarily be increased by a simple enhancement in the wiggle velocity.

For sufficiently smaller values of $\beta_{0}$, group-I orbits appear (see Fig. 11 for $\beta_{0}=0.75$ ) over a limited range of wiggler field amplitudes. The peak gain (as well as the corresponding frequency) for this case is shown in Fig. 12, and describes the frequency and gain associated with the group-I orbits. We have restricted consideration to group-I orbits, because while group-II orbits also lead to growth, the gain is weaker and the frequency is relatively low $\left(\omega \lesssim 8 k_{w} c\right)$. For these orbits, the gain is seen to increase with increasing wiggle velocity, and the singularity mentioned previously is also found at the transition to orbital instability. It should be remarked, however, that the small-signal approximation breaks down in the vicinity of the singularity.

The gain for gyroresonant emission is given by $\left(\omega \simeq \Omega_{0}+k v_{\| 0}\right)$

$$
G_{L} \simeq-\frac{\omega_{b}^{2}}{2 k c^{2}} \frac{L^{2} \Omega_{0}}{\gamma_{0} v_{\| 0}}\left[1+\frac{\beta_{w 0}^{2}}{2}\left[1+\frac{\omega}{k_{w} v_{\| 0}-\Omega_{0}}\right]\right] \frac{\sin ^{2} \theta_{g 0}}{\theta_{g 0}^{2}}
$$

for a long interaction region $\left(k_{w} L>>1\right)$, and growth is possible if $k_{w} v_{\| 0} \lesssim \Omega_{0}$ and

$$
\beta_{w 0}^{2} \geq \frac{\left(\Omega_{0}-k_{w} v_{\| 0}\right)}{\left(k+k_{w}\right) v_{\| 0}}
$$

Peak amplification occurs at the maximum of $\left(\sin \theta_{g 0} / \theta_{g 0}\right)^{2} \simeq 1$, which corresponds to a frequency $\omega=\Omega_{0}+k v_{\| 0}$ and peak gain

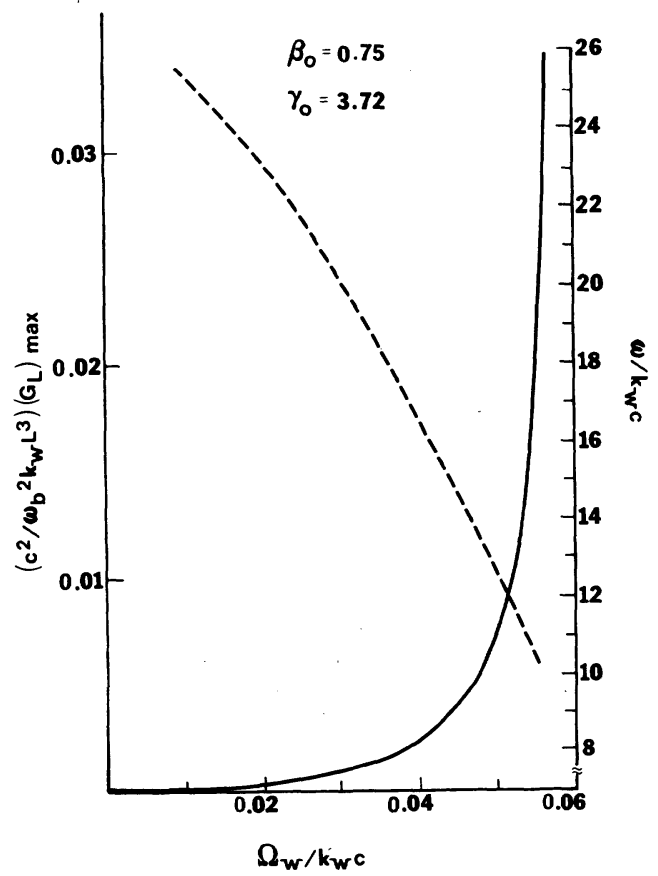

FIG. 12. Graphs of the maximum gain and corresponding frequency for $\beta_{0}=0.75$ and $v / c=0.96$.
$\left(G_{L}\right)_{\max } \simeq \frac{\omega_{b}^{2} L^{2}}{2 \gamma_{0} c^{2}}\left[\frac{\beta_{w 0}^{2}}{2} \frac{\left(1+v_{\| 0} / c\right) \gamma_{z 0}^{2} \Omega_{0}+k_{w} c}{\Omega_{0}-k_{w} v_{\| 0}}-\frac{c}{v_{\| 0}}\right)$.

The variation of the peak gain and associated frequency for gyroresonant emission with axial field strength are shown in Fig. 13 for parameters consistent with the calculation of the frequency and peak gain at the free-electron-laser resonance presented in Fig. 9. Gyroresonant emission is found only for group-II orbits and, as expected, the

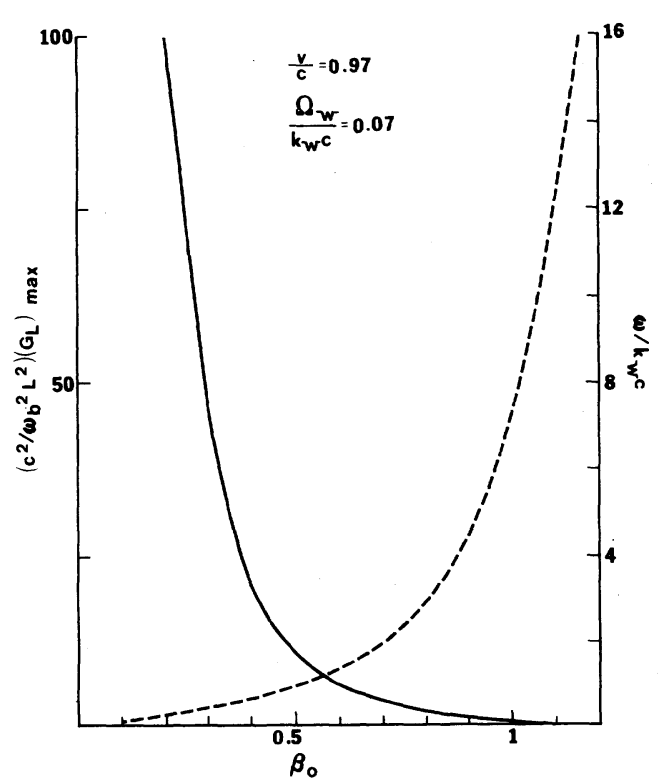

FIG. 13. Graph of the maximum gain and associated frequency versus $\beta_{0}$ for cyclotron emission. 
frequency increases with increasing $\beta_{0}$. In contrast, the gain is found to decrease with increasing axial field strengths. The large increase in the gain as $\beta_{0}$ approaches zero results from the fact that $v_{\| 0}$ also vanishes in this limit. However, the smallsignal-gain approximation breaks down in this limit, and nonlinear effects can be expected to produce enhancements lower than expected on the basis of the linearized theory.

\section{SUMMARY AND DISCUSSION}

In this work, we have considered both the spontaneous and induced emission from a free-electron laser in which a uniform axial guide field is present. The existence of the axial field is found to introduce an additional source of coupling between the axial and transverse electron motion which, for sufficiently strong field levels (44), can lead to orbital instability for motion parallel to $\overrightarrow{\mathrm{B}}_{0}$. Since the existence of orbital instability results in a rapid dispersal of an initially bunched electron beam with disastrous implications for the expected radiation levels, we focus attention on the parameter regimes corresponding to orbital stability and orbits characterized by nearly uniform axial velocities. The predominant transverse motion of these orbits is to track the wiggler field; however, a relatively small component of Larmor rotation associated with the guide field may also be present. Because of this, emission can be expected for both the freeelectron laser and gyrotron resonance conditions.

The spontaneous emission describes the ambient level of noise within the device prior to the coherent amplification process. As shown in Eq. (21), the spontaneous emissivity for waves propagating axially contains components due to the transverse motion resulting from the action of the wiggler and axial guide fields. In each case the level of emission is proportional to the square of the magnitude of the transverse velocities. This velocity corresponds to the canonical momentum in the transverse direction for the gyrotron mode $\left(\omega \simeq \Omega_{0}+k v_{\|}\right)$, and to the wiggler velocity $v_{w}$ for the free-electron-laser mode $\left[\omega \simeq\left(k_{w}+k\right) v_{\|}\right]$. It should be recognized that the presence of a finite $\overrightarrow{\mathrm{B}}_{0}$ leads to an enhancement in $v_{w}$ and, consequent$1 y$, in the free-electron-laser emissivity by a factor of $\left(\Omega_{0} / k_{w} v_{\|}-1\right)^{-2}$. As discussed in Sec. IV, the limit in which $\Omega_{0}=k_{w} v_{\|}$is not achievable.

Enhancement in the linearized (or small-signal) gain are also expected to result when an axial guide field is present. For the tenuous-beam, low-gain regime the enhancement of the gain is expected to be greatest for parameters in the vicinity of the transition to orbital instability (denoted by the dashed line in Fig. 9), which corresponds to a beam characterized by orbital stability. In order for the analysis to be valid for orbitally stable beams, however, stringent requirements on beam quality must be satisfied because a small energy spread can lead to a rapid breakdown in the coher ence of the beam. In addition, the small-signalgain approximation must break down in the vicini. ty of the resonance (i.e., the transition to orbital instability). Finally, significant enhancements in the gain are expected to occur for low-wavelength oscillations only in the low- $\beta_{0}$ regime.

\section{ACKNOWLEDGMENTS}

This work was supported, in part, under NAVAIR Contract No. WF32-389-592. One of us (H.P.F.) would like to thank the Instituto de Fisica of the Universidade Federal do Rio Grande do Sul for hospitality during his visit. In addition, support for several of us (D.D., E.H.J., B.L., and R.S.S.) was provided by Conselho Nacional de Desenvolvimento Cientifico e Technologico (CNPq) and Financiadora de Estudos e Projetos (FINEP).
*Permanent address: Science Applications, Inc., McLean, Virginia 22102.

${ }^{1}$ L. R. Elias, W. M. Fairbank, J. M. J. Madey, H. A. Schwettman, and T. I. Smith, Phys. Rev. Lett. 36, 717 (1976).

${ }^{2}$ D. A. G. Deacon, L. R. Elias, J. M. J. Madey, G. J. Ramian, H. A. Schwettman, and T. I. Smith, Phys. Rev. Lett. 38, 892 (1977).

${ }^{3}$ D. B. McDermott, T. C. Marshall, S. P. Schlesinger, R.
K. Parker, and V. L. Granatstein, Phys. Rev. Lett. 41, 1368 (1978).

${ }^{4}$ P. Sprangle and R. A. Smith, Phys. Rev. A 21, 293 (1980).

5I. B. Bernstein and J. L. Hirschfield, Phys. Rev. A 20, 1661 (1979).

${ }^{6}$ R. C. Davidson and H. S. Uhm, Phys. Fluids 23, 2076 (1980).

${ }^{7}$ T. Kwan and J. M. Dawson, Phys. Fluids 22, 1089 
(1979).

${ }^{8}$ M. Zales Caponi, J. Munch, and M. Boehmer, in FreeElectron Generators of Coherent Radiation, edited by S. F. Jacobs, H. S. Pilloff, M. Sargent, M. O. Scully, and R. Spitzer (Addison-Wesley, New York, 1980), p. 523.

${ }^{9}$ L. Friedland and J. L. Hirschfield, Phys. Rev. Lett. 44, 1456 (1980).

10I. B. Bernstein and L. Friedland, Phys. Rev. A $\underline{23}, 816$ (1981).

${ }^{11}$ L. Friedland and J. L. Hirschfield, Phys. Rev. Lett. 44, 1456 (1980).

12J. P. Blewitt and R. Chasman, J. Appl. Phys. $\underline{48}$, 2692 (1977).

${ }^{13}$ P. Diament (unpublished).
${ }^{14}$ R. C. Davidson and H. S. Uhm (unpublished).

${ }^{15}$ H. P. Freund and A. T. Drobot, Phys. Fluids (in press).

${ }^{16} \mathrm{~L}$. Friedland, Phys. Fluids 23, 2376 (1980).

${ }^{17}$ R. E. Shefer and G. Bekefi, Bull. Am. Phys. Soc. 25, 911 (1980).

18J. M. Buzzi, K. Fetch, and L. Vallier, Bull. Am. Phys. Soc. 25,887 (1980).

${ }^{19}$ V. P. Sukhatme and P. W. Wolfe, J. Appl. Phys. 44, 2331 (1973).

20W. B. Colson, Phys. Lett. 59A, 187 (1976).

${ }^{21}$ F. A. Hopf, P. Meystre, M. O. Scully, and W. H. Louisell, Opt. Commun. 18, 413 (1976).

${ }^{22}$ N. M. Kroll and W. A. McMullin, Phys. Rev. A $\underline{17}$, 300 (1978). 